\title{
Multi-Dimensional Hybrid Fourier Continuation-WENO Solvers for Conservation Laws
}

\author{
Khosro Shahbazi ${ }^{\mathrm{a}, *}$, Jan S. Hesthaven ${ }^{\mathrm{b}}$, Xueyu Zhu ${ }^{\mathrm{b}}$ \\ ${ }^{a}$ Mechanical Engineering, South Dakota School of Mines \& Technology, 501 E. St. Joseph St., Rapid City, SD 57r01, United \\ States \\ ${ }^{b}$ Division of Applied Mathematics, Brown University, 182 George Street, Providence, RI 02912, United States
}

\begin{abstract}
We introduce a multi-dimensional point-wise multi-domain hybrid Fourier-Continuation/WENO technique (FC-WENO) that enables high-order and non-oscillatory solution of systems of nonlinear conservation laws, and essentially dispersionless, spectral, solution away from discontinuities, as well as mild CFL constraints for explicit time stepping schemes. The hybrid scheme conjugates the expensive, shock-capturing WENO method in small regions containing discontinuities with the efficient FC method in the rest of the computational domain, yielding a highly effective overall scheme for applications with a mix of discontinuities and complex smooth structures. The smooth and discontinuous solution regions are distinguished using the multi-resolution procedure of Harten [J. Comput. Phys. 115 (1994) 319-338]. We consider a WENO scheme of formal order nine and a FC method of order five. The accuracy, stability and efficiency of the new hybrid method for conservation laws are investigated for problems with both smooth and non-smooth solutions. The Euler equations for gas dynamics are solved for the Mach 3 and Mach 1.25 shock wave interaction with a small, plain, oblique entropy wave using the hybrid FC-WENO, the pure WENO and the hybrid central difference-WENO (CD-WENO) schemes. We demonstrate considerable computational advantages of the new FC-based method over the two alternatives. Moreover, in solving a challenging two-dimensional Richtmyer-Meshkov instability (RMI), the hybrid solver results in seven-fold speedup over the pure WENO scheme. Thanks to the multi-domain formulation of the solver, the scheme is straightforwardly implemented on parallel processors using message passing interface as well as on Graphics Processing Units (GPUs) using CUDA programming language. The performance of the solver on parallel CPUs yields almost perfect scaling, illustrating the minimal communication requirements of the multi-domain strategy. For the same RMI test, the hybrid computations on a single GPU, in double precision arithmetics, displays five- to six-fold speedup over the hybrid computations on a single CPU. The relative speedup of the hybrid computation over the WENO computations on GPUs is similar to that on CPUs, demonstrating the advantage of hybrid schemes technique on both CPUs and GPUs.
\end{abstract}

Keywords: Fourier continuation Methods, high-order WENO methods, multi-resolution methods, conservation laws, shock waves, parallel and many core computation

\section{Introduction}

Solutions of high-speed flow problems are often characterized by a challenging combination of sharp gradients, discontinuities and regions of complex but smooth flow structures; examples of such situations are provided by the well known shock-induced multi-material flow instabilities, mixing, and acoustic noise generation by turbulent flows. To accurately capture all regimes in such complicated flow it is necessary to account accurately for both sharp nonlinear discontinuities as well as complex smooth flow structures. While it is well known that high-order accurate methods, e.g high-order finite difference methods or spectral

\footnotetext{
* Corresponding author.

Email addresses: khosro.shahbazi@sdsmt.edu (Khosro Shahbazi), jan.hesthaven@brown.edu (Jan S. Hesthaven), xueyu.zhu@brown.edu (Xueyu Zhu)
} 
methods, are well suited for the smooth elements of the solution, it is also well appreciated that such techniques introduce oscillatory behavior near discontinuities [20, 21].

A well-known alternative for the solution of such flows is based on a high-order weighted essentially nonoscillatory (WENO) finite difference method; in particular, high-order WENO algorithms have been used to produce successful simulations of the Rayleigh-Taylor instability [24] and Richtmyer-Meshkov instability [25, $26]$ in two and three space dimensions. Finite volume version of the high-order WENO scheme have been used in shock wave bubble interactions in three dimensions [36]. It is notable that although the formal high-order accuracy of the WENO schemes is degraded near discontinuities and large solution gradients, the high-order scheme do indeed achieve higher level of accuracy/resolution in a more computational efficient fashion as demonstrated by recent investigations [24, 25,39]. Recent research activities in extending and optimizing WENO schemes to orders up to $16[37,38]$ may also be motivated by the computational efficiency of these higher order schemes over their lower order counterparts.

Unfortunately, the WENO finite difference methods are generally computationally very expensive and do not compare favorably with linear schemes such as finite difference or spectral methods. The reason for this is at least two-fold. The WENO schemes are, on one hand, more expensive based on operation counts per grid point, often by a constant factor of roughly ten in three dimensions. This is due to several costly WENO operations: the characteristic decomposition of fluxes to control oscillations, the calculation of nonlinear weights both for smooth and non-smooth flux contributions, and double operations for "positive" and "negative" flux splits. Secondly, the WENO scheme requires a substantial and increasing number of grid points to achieve a specific accuracy level as the solution frequency spectrum widens, or as spatial domains are enlarged and larger physical simulation time are sought. This is due to the upwinding nature of the WENO schemes essential for non-oscillatory capturing of discontinuities. In view of the complexity of threedimensional computations of complex high-speed flows, it is crucial to seek a more efficient alternative to enable the ability to model complex problems and multi material scenarios.

We focus on problems in which the solution discontinuities are mainly local phenomena in both space and time. This suggests that a hybrid scheme could advantageously be used whereby the "expensive" WENO procedure is only used in parts of the space-time domain containing discontinuities, while a less costly, highorder linear numerical method is employed for the portion of the domain containing complex but smooth features. For problems with localized shocks and extended regions of mixing or acoustic noise propagation, we would expect such an approach to yield a considerably faster algorithm.

The core of such a hybrid approach is the use of an efficient numerical method for smooth regions together with an effective "smoothness-indicator" strategy to identify areas of smoothness and discontinuity. For the former, a central difference (upwind-biased or unbiased) or a Chebyshev-based spectral methods are possible choices that have been explored previously, e.g., in [10,12] a hybridization of spectral Chebyshev and WENO methods for conservation laws in one and two space dimensions is proposed and applied to a complex simulation of early-stage two-dimensional Richtmyer-Meshkov instability while a hybrid central differenceWENO scheme in one space dimension is presented in [11]. Both of these developments accurately identify discontinuities using the multi-resolution algorithm of Harten [9], based on differences of the point-values of a function and its high-order interpolated function on coarser grids $[10,12]$.

The advantage of the very high order Chebyshev spectral methods over the central difference methods lies in the higher order of accuracy near the non-periodic boundaries and, perhaps of most importance, solutions free of dispersion error (also known as pollution error). Absence of dispersion error is crucial for efficient simulation of problems with a wide range of spatial and temporal scales including transitional and turbulent flows [28].

As we shall discuss shortly, the hybrid scheme proposed here maintains these properties while overcoming some severe restrictions imposed by the scheme based on Chebyshev polynomials. A particular consequence of the use of the Chebyshev spectral method is the presence of grid points that cluster near the boundaries of each such sub-domain, impacting the overall performance of the scheme in different ways. On one hand, the WENO finite difference method is defined on an equidistant grid. Thus, the two grids in a Chebyshev-WENO hybrid do not conform in any natural manner, implying that extra interpolation operations are required for transfer of data at overlapping regions between adjacent domains with two different discretization schemes. This also complicates the multi-resolution analysis. Furthermore, for explicit time integration strategies, a 
hybrid Chebyshev-WENO method requires a much smaller $\left(\mathcal{O}\left(1 / N^{2}\right)\right)$ time step than a scheme based on an equidistant grid due to the nature of the polynomial approximation, leading to a very stringent time-step restriction that is particularly problematic if long time integration and/or very high accuracy is required.

In this work, extending the one-dimensional version of the hybrid approach [39] to two-dimensional problems, we propose an attractive alternative in which we hybridize the WENO method with a recently proposed Fourier continuation (FC) method [1,6, 7]. The FC approximation is based on a high-order periodic continuation of a (possibly non-periodic) function, yet being based on a Fourier method, the FC approximation has almost no dispersion (pollution) error and, utilizing an equispaced grid, provides a simple and efficient interface with WENO scheme and the multi-resolution analysis while allowing for an efficient temporal integration as compared to the Chebyshev-WENO scheme. At high resolution it furthermore allows for the use of the Fast Fourier Transform, enabling a further acceleration.

The remainder of the paper is organized as follows. In Sec. 2 we review the Fourier continuation (FC) method for the approximation of functions, and in Sec. 3 we discuss our multi-domain FC-WENO hybrid method for two-dimensional conservation laws. This sets the stage for Sec. 4 where we present a variety of numerical results for problems governed by the inviscid Burgers' equation and the Euler equations. Among other things, we demonstrate the accuracy of the FC method for the Burgers' problems with smooth solutions in both single-domain and multi-domain formulations, and we assess the overall efficiency of our method in the context of challenging computational problems - including nonlinear systems of conservation laws with discontinuous solutions. For Ritchmyer-Meshkov instability problem we demonstrate the speedup of the hybrid scheme over the pure WENO scheme, and also show the performance of the solver on contemporary parallel computers as well as on many core processing units exemplified by Graphic Processing Units (GPUs). In Sec. 5 we offer a few remarks and suggested directions of future research.

\section{The Point-wise Fourier continuation approximation}

The point-wise, as opposed to cell-averaging, nature of the FC approximation considered here allows us to compute approximations in multiple dimensions based on a sequence of one-dimensional approximations along lines of points. Thus the one-dimensional FC approximation described in [39] directly applies and we highlight the central elements of this approach below.

Given a (typically non-periodic) smooth function $f$ defined over the interval $(0,1)$ and with values of $f$ given at an $N$-point equi-spaced grid $\left\{x_{k}\right\}_{k=0}^{N-1} \subseteq(0,1)$ purely internal to the domain, the Fourier continuation (extension) method seeks to produce, on the basis of $\left\{f\left(x_{k}\right)\right\}_{k=0}^{N-1}$, a periodic function $\tilde{f}$ expressed by a finite number $M$ of Fourier modes,

$$
\tilde{f}(x)=\sum_{j \in g(M)} \hat{a}_{j} e^{\left(\frac{2 \pi i}{1+d} j x\right)} .
$$

and defined over a larger domain $(0,1+d)$ in such a way that it matches closely the original function $f$ throughout the original interval $(0,1)$. Here $g(M)=\{j \in \mathbb{N} \mid-M / 2+1 \leq j \leq M / 2\}$ for $M$ even and $g(M)=\{j \in \mathbb{N} \mid-(M-1) / 2 \leq j \leq(M-1) / 2\}$ for $M$ odd. In general, $N$ must be chosen larger than $M$ due to the intrinsic ill-conditioning of the resulting linear system; as shown in $[2,5]$, the least-squares solution via singular value decomposition of the over-determined system

$$
\sum_{j \in g(M)} \hat{a}_{j} e^{\left(\frac{2 \pi i}{1+d} j x_{k}\right)}=f\left(x_{k}\right) \quad \forall k=0, \cdots, N-1,
$$

for the coefficients $\hat{a}_{j}$ leads to an effective Fourier Continuation strategy.

However, it is observed that a smooth and accurate continuation of $f(x)$ does not necessarily require the value of the function over the entire interval $(0,1)$ and only the function values on small intervals near the boundaries (boundary intervals) are sufficient. A continuation method based on this philosophy requires finding a matching function $\tilde{h}(x)$ defined over $(1-\Delta, 1+2 d+\Delta)$ with periodicity $2 d+2 \Delta$ as

$$
\tilde{h}(x)=\sum_{j \in g(M)} \hat{a}_{j} e^{\left(\frac{\pi i}{d+\Delta} j x\right)},
$$




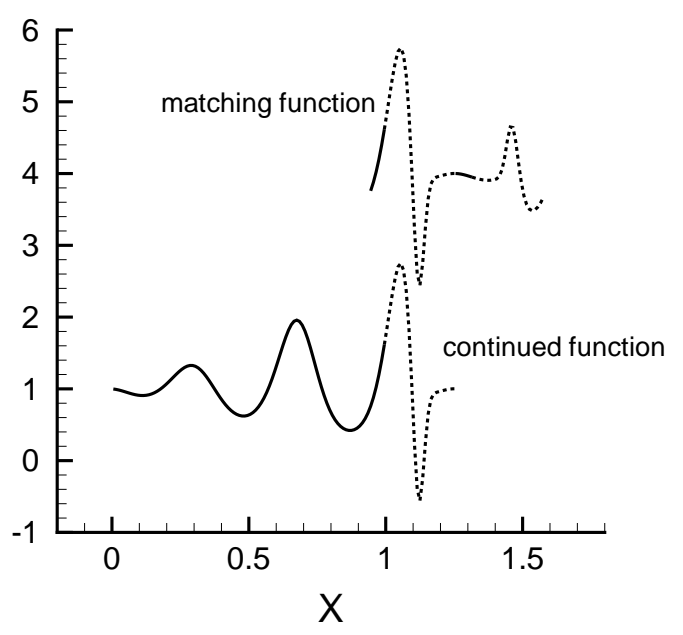

Figure 1: Continuation of $f(x)=\exp (x \sin (16(x+1))), x \in(0,1)$, based on Eq. 5, the continuation interval $d=0.26$ and boundary interval size $\Delta=0.06$. In the lower diagram, the original function and its continuation are shown in solid and dotted line, respectively; In the upper diagram, the matching function $h(x)$ is shown, raised three units for better visibility. In the upper diagram, the two solid line segments mark values of $\tilde{h}(x)$ matching $f(x)$ at the two boundary intervals $(0, \Delta)$ and $(1-\Delta, 1)$.

$\tilde{h}(x)$ matches the original function $f(x)$, in the least squares sense, as

$$
\begin{aligned}
\tilde{h}\left(z_{k}+1-\Delta\right) & =f\left(z_{k}+1-\Delta\right) \\
\tilde{h}\left(1+d+z_{k}\right) & =f\left(z_{k}\right)
\end{aligned}
$$

for a sufficiently large fine equi-spaced grid points $z_{k}$ defined over $(0, \Delta)$. The Fourier continuation of $f(x)$, $\tilde{f}(x)$, is then obtained as

$$
\begin{array}{ll}
\tilde{f}(x)=f(x) & 0 \leq x \leq 1, \\
\tilde{f}(x)=\tilde{h}(x) & 1<x \leq 1+d .
\end{array}
$$

Fig. 1 illustrates the basic idea behind the continuation and the matching function for a sample function.

As an alternative approach to decrease the impact of the ill-conditioning without incurring the high $\mathcal{O}\left(N^{3}\right)$ computational cost arising from the evaluation of the singular value decomposition [8], Bruno and Lyon [6] proposed a different strategy based on the use of Gram polynomials [30]. Their approach, also adopted here, uses the continuation of Gram polynomials to approximate the matching function $\tilde{h}$. For a given approximation order the set of Gram polynomials are unique and thus their continuations can be carried out in a preprocessing stage and used to approximate the periodic extension of any given function $f$ via its matching function $\tilde{h}$ as detailed in $[6,39]$.

Remark 1. For our numerical examples we have taken the maximum Gram polynomial degree as $p=5$, the number of boundary points $\gamma=p+1=6$, and $d / \Delta=26 / 6$.

It is worth emphasizing that extensive experimentation with higher values of $p$ does not show significant advantages for the class of problems considered in this work. This is mainly due to the limitations in accuracy imposed by the WENO techniques for problems with discontinuous solutions. However, for problems with large regions of smooth solution, improvements may be achieved by increasing this value ([1]).

Remark 2. It may seem counter intuitive that the high-order projection of a function into a polynomial spaces defined by a set of equi-spaced grid points does not exhibit the Runge phenomenon as the approximation approaches the boundary. The explanation for this is that since the polynomial order is kept constant 
- as the number of points per domains increases, the boundary interval shrinks and so does the Runge zone in the complex plane. For details and rigorous proofs of related subjects, we refer to $[3,4]$.

Remark 3. It is interesting to note that, while the polynomial projection of the original function implies a finite order of approximation of the method, the Fourier continuation method retains all the desirable properties normally associated with a Fourier spectral method, e.g., a differential operator with spectrum that scales linearly with the spatial resolution and, as we shall demonstrate in Sec. 4, no pollution error in the sense that for a certain accuracy level, the required number of points per wave length remains constant as the wave number of the solution increases.

\section{Multi-domain Fourier-Continuation/WENO hybrid method}

In the following we describe the four main elements of our multi-domain hybrid FC-WENO method for solution of conservation laws: (1) use of the FC method for derivative computations for the smooth portions of the solutions; (2) the WENO method for regions with steep gradients or discontinuous solutions; (3) the smoothness indicator, which allows us to determine when to switch between the two techniques; (4) a multi-domain formulation ensuring stable and non-oscillatory transfer of data between adjacent domains. For the temporal discretization, we use a standard third-order total variation diminishing (TVD) Runge-Kutta method [29].

\subsection{FC method for conservation laws with smooth solutions}

We consider smooth solutions to a two-dimensional nonlinear scalar conservation law of the form

$$
\frac{\partial u(t, \mathbf{x}))}{\partial t}+\frac{\partial F(u(t, \mathbf{x}))}{\partial x}+\frac{\partial G(u(t, \mathbf{x}))}{\partial y}=0,
$$

defined on a two-dimensional spatial domain $\mathbf{x}=[x, y] \in \Omega=\left[X_{1}, X_{2}\right] \times\left[Y_{1}, Y_{2}\right]$ in the time interval $[0, T]$, subject to appropriate initial and boundary conditions. Given a set of equi-spaced grid points in each direction, $\mathbf{x}_{k}=\left[x_{k}, y_{k}\right]$ defined as $x_{k}=X_{1}+(k+1 / 2) L_{x} / N, k=0, \cdots N-1$, with $L_{x}=X_{2}-X_{1}$, and $y_{k}=Y_{1}+(k+1 / 2) L_{y} / N, k=0, \cdots N-1$, with $L_{y}=Y_{2}-Y_{1}$ we seek $u_{h}\left(t, \mathbf{x}_{k}\right)$, an approximation to $u\left(t, \mathbf{x}_{k}\right)$, that satisfies the equation in a collocation sense

$$
\frac{\partial u_{h}\left(t, \mathbf{x}_{k}\right)}{\partial t}+\frac{\partial u_{h}\left(t, \mathbf{x}_{k}\right)}{\partial x} \frac{\partial F\left(u_{h}\left(t, \mathbf{x}_{k}\right)\right)}{\partial u}+\frac{\partial u_{h}\left(t, \mathbf{x}_{k}\right)}{\partial y} \frac{\partial G\left(u_{h}\left(t, \mathbf{x}_{k}\right)\right)}{\partial u}=0 \quad \forall k=0, \cdots, N-1 .
$$

Note that we have not expressed our equation in conservation form, since (7) leads to a more efficient algorithm in the smooth-region.

The spatial derivatives in the $x$ - and $y$-direction, $\frac{\partial u_{h}\left(t, \mathbf{x}_{k}\right)}{\partial x}$ and $\frac{\partial u_{h}\left(t, \mathbf{x}_{k}\right)}{\partial y}$, are computed using the onedimensional Fourier continuation of the numerical solution along solution data lines in the $x$ - and $y$-direction, respectively. Specifically, using (2), we have the Fourier continuation expansion of $u_{h}\left(t, x, y_{k}\right)$ and $u_{h}\left(t, x_{k}, y\right)$,

$$
\begin{aligned}
& u_{h}\left(t, x, y_{k}\right)=\sum_{j \in g(M)} \hat{a}_{j}\left(t, y_{k}\right) e^{\left(\frac{2 \pi i}{L_{d x}} j x\right)}, \quad \forall k=0, \cdots, N-1, \\
& u_{h}\left(t, x_{k}, y\right)=\sum_{j \in g(M)} \hat{a}_{j}\left(t, x_{k}\right) e^{\left(\frac{2 \pi i}{L_{d y}} j y\right)}, \quad \forall k=0, \cdots, N-1,
\end{aligned}
$$

with $L_{d x}=L_{x}+d$ and $L_{d y}=L_{y}+d$. Thus the spatial derivatives are computed as

$$
\begin{array}{ll}
\frac{\partial u_{h}\left(t, x, y_{k}\right)}{\partial x}=\sum_{j \in g(M)} \frac{2 \pi i j}{L_{d x}} \hat{a}_{j}\left(t, y_{k}\right) e^{\left(\frac{2 \pi i}{L_{d x}} j x\right)}, \quad \forall k=0, \cdots, N-1, \\
\frac{\partial u_{h}\left(t, x_{k}, y\right)}{\partial y}=\sum_{j \in g(M)} \frac{2 \pi i j}{L_{d y}} \hat{a}_{j}\left(t, x_{k}\right) e^{\left(\frac{2 \pi i}{L_{d y}} j y\right)}, \quad \forall k=0, \cdots, N-1,
\end{array}
$$


As discussed in details in [39], the appearance of very small unstable eigenvalues through the Fourier continuation may render this approach weakly unstable. To remedy this, a very weak exponential filter is used to damp out high-frequency modes with minimal adverse impact on accuracy [20]. For number of grids points per domain and per direction of $N P$, the filter order is typically chosen to be $N P / 2$. Details of the exponential filter and the boundary condition imposition are identical to the one-dimensional case and can be found in [39].

Remark 4. For nonlinear problems, the use of an exponential filter serves the additional purpose of removing energy accumulated at high frequencies, and thus overcoming instabilities due to the nonlinearity.

Remark 5. Note that with $p=5$ in the FC approximation, the derivative computation is technically fifth-order accurate, i.e., our FC method for the conservation law (6) with smooth solution is formally fifthorder accurate. However, as we discuss shortly, the behavior of the method away from the boundaries is closer to that of a Fourier spectral method and the performance is not impacted by the nominal fifth order of approximation.

Remark 6. Regarding the implementation of the derivative calculations, the Fourier coefficients in (8a or 8b) are first computed using a fast Fourier transform (FFT) and point-values of the derivatives in (9a or 9b) are obtained using an inverse FFT. Both steps, FFT and inverse FFT, can be computed with a complexity of $\mathcal{O}(N \log (N))$. Alternatively, the derivative calculation can be performed using a matrix-vector product with a cost of $\mathcal{O}\left(N^{2}\right)$ with the matrix representing the effect of differentiation and the vector being the collocation values of the function [21]. For larger values $N$, the former is clearly preferred and that is the approach we consider here.

Remark 7. Equation (7) is on non-conservative form. The equivalent conservative form (for smooth problems) is

$$
\frac{\partial u_{h}\left(t, \mathbf{x}_{k}\right)}{\partial t}+\frac{\partial F\left(u_{h}\left(t, \mathbf{x}_{k}\right)\right)}{\partial x}+\frac{\partial G\left(u_{h}\left(t, \mathbf{x}_{k}\right)\right)}{\partial y}=0 \quad \forall k=0, \cdots, N-1 .
$$

In all our numerical tests, both conservative and non-conservative FC discretizations of the conservation laws have been tested and observed to yield almost identical results. This is natural since the FC approach is only used in regions with smooth solutions. Our preference for the non-conservative form is guided by efficiency. While the non-conservative form requires only the continuation of $u$ and its filtering, the conservative form requires continuation and filtering of both $u$ and the flux, $f(u)$ and, thus, renders the scheme more expensive.

\subsection{WENO Methods for conservation laws with discontinuous solutions}

WENO finite difference methods are finite difference schemes tailored to enable the accurate solution of conservation laws involving discontinuous solutions - or, more generally, solutions which contain steep/unresolved gradients. The details of the WENO schemes are well-documented [18, 15], and we therefore offer only a brief presentation of the essential elements of these methods.

A traditional spatial finite difference discretization of (6) seeks point-wise enforcement of the equation at a set of equi-spaced grid points in each direction $x_{i}=X_{1}+(i+1 / 2) L_{x} / N, y_{i}=Y_{1}+(i+1 / 2) L_{y} / N$ $i=0, \cdots N-1$, as

$$
\frac{\left.\partial u_{h}\left(t, \mathbf{x}_{i}\right)\right)}{\partial t}+\frac{\hat{F}\left(t, x_{i+1 / 2}, y_{i}\right)-\hat{F}\left(t, x_{i-1 / 2}, y_{i}\right)}{\Delta x}+\frac{\hat{G}\left(t, x_{i}, y_{i+1 / 2}\right)-\hat{G}\left(t, x_{i}, y_{i-1 / 2}\right)}{\Delta y}=0,
$$

where $\Delta x=x_{i}-x_{i-1}, \Delta y=y_{i}-y_{i-1}, u_{h}\left(t, x_{i}\right)$ is a numerical approximation of $u\left(t, \mathbf{x}_{i}\right)$ and where $\hat{F}\left(t, x_{i+1 / 2}, y_{k}\right)\left(\hat{F}\left(t, x_{i-1 / 2}, y_{i}\right)\right)$ and $\hat{G}\left(t, x_{i}, y_{i+1 / 2}\right)\left(\hat{G}\left(t, x_{i}, y_{i-1 / 2}\right)\right)$ represent numerical fluxes computed using the solution data along the $x$ - and $y$-direction respectively. The numerical flux $\hat{f}_{i+1 / 2}$ is defined through a reconstruction based on the numerical solutions at $x_{i}$ (or $y_{i}$ ), $r$ solution to the left (or south) and $s$ solutions to the right (or north) of $x_{i}$ (or $y_{i}$ ):

$$
\hat{f}_{i+1 / 2}=\hat{f}\left(u_{i-r}, \cdots, u_{i+s}\right) .
$$


Here $\hat{f}$ is required to be Lipschitz continuous in all arguments and consistent with the physical flux $f$, that is, $\hat{f}(u, \cdots, u)=f(u)$. Based on the Lax-Wendroff theorem, the solution of this conservative scheme, if it converges, converges to the weak solution of (6).

The precise choice of the numerical flux $\hat{f}_{i+1 / 2}$ is central and must follow a number of guidelines. To guarantee entropy dissipation, the flux is first split into positive and negative parts using Lax-Friedrichs splitting as $f(u)=f^{+}(u)+f^{-}(u)$, with $f^{+}(u)=(f(u)+\alpha u) / 2, f^{-}(u)=(f(u)-\alpha u) / 2$, and $\alpha=\max _{u} \frac{d f(u)}{d u}$. The reconstruction is applied for each positive and negative flux separately, before adding up to give the numerical flux.

A finite difference reconstruction of the flux in the $x$-direction or the $y$-direction, for instance for $f^{+}\left(u_{i+1 / 2}\right)$, can be computed using solutions in $k$ different substencils,

$$
\mathcal{S}_{k}(i)=x_{i-r}, \cdots, x_{i-r+k-1} \quad r=0, \cdots, k-1
$$

or

$$
\mathcal{S}_{k}(i)=y_{i-r}, \cdots, y_{i-r+k-1} \quad r=0, \cdots, k-1,
$$

as

$$
\hat{f}_{r}^{+}\left(u_{i+1 / 2}\right)=\sum_{j=0}^{k-1} C_{r, j} f^{+}\left(u_{i-r+j}\right) .
$$

The constant coefficients $C_{r, n}$ (given in [14] up to order seven) are chosen such that the approximate solution is formally accurate up to order $k$ in regions in which the exact solution is sufficiently smooth. On the other hand, if in a stencil, the solution or one of its $j$-derivatives $j \leq q$ contains a discontinuity, that stencil must be excluded from the flux approximation. This nonlinear procedure is referred to as an essentially non-oscillatory (ENO) scheme [17]. A slightly modified approach, termed weighted essentially nonoscillatory (WENO), offering computational advantages and added robustness (see [14]), is to consider a convex combination of all computed fluxes $f_{r}^{+}\left(u_{i+1 / 2}\right)$ in $k$ stencils as

$$
\hat{f}_{w}^{+}\left(u_{i+1 / 2}\right)=\sum_{r=0}^{k-1} w_{r} \hat{f}_{r}^{+}\left(u_{i+1 / 2}\right),
$$

where $w_{r}$ is chosen such that the approximation is of order $2 k-1$ accuracy in cases where the solution is sufficiently smooth in all stencils. An explicit expression for $w_{r}$, based on a local smoothness indicator for orders $k=2$, and 3, are given in [13] and for higher orders up to $k=6$ in [16]. The WENO parameters $\epsilon$ and $p$ are chosen to be $10^{-6}$ and 2 , respectively [15]. In the following we use the ninth-order variant, introduced in reference [16].

Remark 8. For system of conservation laws, such as the Euler equations, it is essential to apply the WENO reconstruction to the fluxes in the characteristic variables. This is enabled through multiplication of the flux vectors with the left eigensystem. The reconstructed fluxes are then transferred back to the conservative fluxes through multiplication with the right eigensystem [13]. Both left and right eigensystems are evaluated using Roe-averaged field variables.

\subsection{Detecting the discontinuities}

Detecting discontinuities in the solution or its derivatives is an essential component and prerequisite for the hybrid strategy to be effective. For systems of nonlinear conservation laws like the Euler system of gas dynamics, the location of the discontinuities, e.g. shocks, are not known a priori and may emerge and propagate as the solution advances. We thus need to estimate the local smoothness from the solution field at regular temporal intervals. To this end, the multi-resolution (MR) analysis introduced by Harten [9] and later used in the context of a hybrid spectral-WENO method by Costa and Don [10] has proven to be effective in the one-dimension hybrid FC-WENO approach. We extend the approach to two dimensions by applying the MR analysis line by line first in the $x$-direction and then in the $y$-direction. A brief description of this approach is given below; a detailed account can be found in $[9,10]$. 
Using the solution values $f_{k}^{0}$ at equi-spaced grid points $x_{k}^{0}(k=1, \cdots, 2 N+1)$ at which the approximate solutions are known, we construct the solution averages $f_{k}^{1}$ at one level coarser grid points $x_{k}^{1}=\left(x_{2 k-1}^{0}+\right.$ $\left.x_{2 k+1}^{0}\right) / 2$ with $k=1, \cdots, N$ as

$$
f_{k}^{1}=\frac{f_{2 k}^{0}+f_{2 k+1}^{0}}{2} \quad \forall k=1, \cdots, N .
$$

Let $p_{s}(x)$ denote the polynomial approximation of order $s$ interpolating $f_{k}^{1}$ at the coarser grid points $x_{k}^{1}$. The approximation differences, $d_{k}=f_{k}^{0}-p_{s}\left(x_{k}^{0}\right)$, have the property that if $f(x)$ has $r-1$ continuous derivatives and a jump discontinuity at $r$ th derivative, then

$$
d_{k}=\left\{\begin{array}{cl}
\Delta x^{r}\left[\frac{d^{r} f_{k}}{d x^{r}}\right] & s \geq r \\
\Delta x^{s} \frac{d^{s} f_{k}}{d x^{s}} & s<r
\end{array}\right.
$$

where $\Delta x$ denotes the coarse grid spacing and [.] denotes jumps across a discontinuity. This implies that the higher degree of smoothness of the solution, and the higher the order of the polynomial approximation on the coarser grid (in the case of smooth function), the smaller the approximation differences, $d_{k}$. We thus adopt a tolerance $\epsilon$, below which the function is assumed to be smooth and otherwise identify it as nonsmooth. Once we have found the approximate locations of the discontinuities by this method, the domains containing smooth solutions are treated by the FC method and those with non-smooth solutions are treated by the WENO scheme. This simple strategy works well except for situations in which a discontinuity is very close to a boundary of a sub-domain, within a few grid points. In this case, the neighboring domain is also marked as a WENO domain to ensure conservative and non-oscillatory transfer of the discontinuity between neighboring domains. This strategy was previously adopted in $[10,12]$. In our implementation, the detection of a discontinuity within two grid points away from a sub-domain boundary dictates the WENO treatment of the sub-domain.

\subsection{Point-wise multi-domain formulation}

To accommodate the distinct treatment of smooth solution regions and discontinuous regions, we pursue a multi-domain solution algorithm. The computational domain is first decomposed into sub-domains with each sub-domain being discretized with either an FC or a WENO method depending on the local smoothness indication. A domain interface condition is adopted for stable and non-oscillatory transfer of data between adjacent sub-domains. The decomposition of the domain can be performed using two different strategies: (1) single decomposition, resulting sub-domains with fixed sizes and with fixed number of grid points, or (2) repeated decomposition at regular intervals of the time marching yielding sub-domains with time-varying sizes, and/or with number of grid points varying in time. We adopt the fixed-sized domain strategy since it facilitates the extension of our multi-domain strategy to relatively complex geometries in multiple space dimensions in a straightforward block structured manner. Figs. 2 (a) and (b) demonstrate a two-dimensional domain decomposition of a square domain into four subdomains. Also shown in the figures are grid points which are internal to each sub-domains.

To ensure a stable approach for transfer of data between adjacent sub-domains, we utilize an overlapping grid stencil. Specifically, for an internal sub-domain marked as a smooth region, a derivative computation in the $x$ - or $y$-direction for all points along a line of points based on the FC method is carried out using an extended stencil consisting of the sub-domain's own grid points along the same line and a few of the closest grid points of its neighbors (Fig. 2 (b)). For a boundary domain, the extension is one-sided across the internal interface (Fig. 2 (b)). Using this process, the derivatives of the solution are available for the extended stencil. However, only the computed derivatives of the actual sub-domain grid points are used for updating the solution field, and derivatives at the extended grid points are discarded. Extensive experimentation confirms that an extension of three grid points across each domain interface suffices to guarantee stability. For WENO sub-domains, a similar strategy is required with extensions of three and five grid points in each direction for fifth- and ninth-order schemes, respectively (Fig. 2 (b)).

Note that this strategy of handling or circumventing interface conditions is uniformly valid regardless of whether adjacent domains are two FC domains, or two WENO domains, or one FC and one WENO. This is a consequence of using equi-spaced internal grid points (not including end points, see Fig. 2 (b)) for both FC and WENO discretizations. 


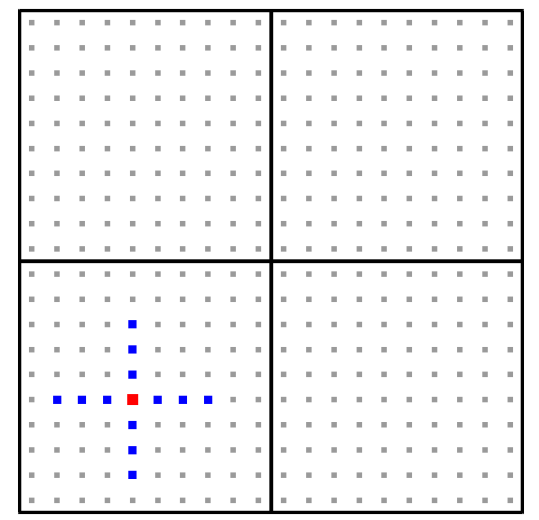

(a)

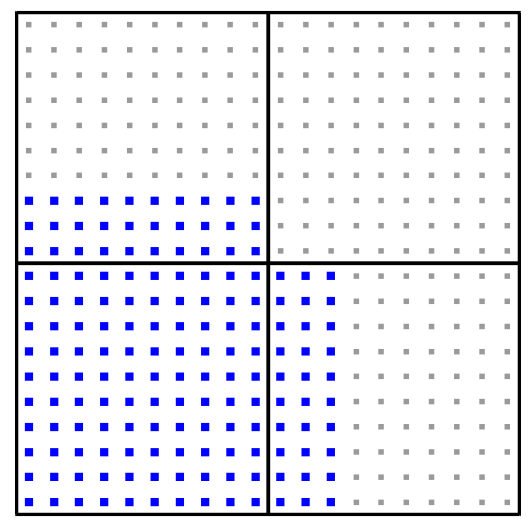

(b)

Figure 2: Point-wise multi-domain formulations; (a) A local stencil, consisting of the blue squares and the red square in horizontal or vertical directions used to compute flux derivatives in horizontal or vertical direction using a fifth-order WENO finite difference reconstruction; (b) A global extended stencil containing of grid points in blue for computing derivatives in horizontal or vertical directions for all points along a horizontal or vertical direction using Fourier continuation method.

\section{Numerical Examples}

In this section we strive to carefully and rigorously evaluate all elements of the proposed algorithm. We first verify the performance of the high order scheme for solving the inviscid Burgers' equation in two dimensions. We continue by considering a number of results for the Euler system where an extensive set of comparisons of our hybrid FC-WENO method with pure WENO and another hybrid scheme arising from conjugation of the central difference and WENO schemes in solving shock-entropy-wave-interaction problems. Finally, we show the performance of the FC-WENO scheme in solving a two-dimensional Richmyer-Meshkov instability problem as well as its performance on parallel processors and emerging many core Graphics Computing Units (GPUs). As we shall see, all cases demonstrate substantial advantages over existing alternatives, both in terms of accuracy and computational performance.

\subsection{D Inviscid Burgers' equation}

As a first test, we consider a 2D inviscid Burgers' equation (also known as the Riemann equation) in the form of

$$
\frac{\partial u}{\partial t}+\frac{\partial u^{2} / 2}{\partial x}+\frac{\partial u^{2} / 2}{\partial y}=0
$$

The computational domain is $[-1,1] \times[-1,1]$ with periodic boundary conditions in both directions and the initial condition is taken to be $u(0, x)=(1+\sin (\pi(x+y))) / 2$. Using the characteristic method, the exact solution of this equation is given as

$$
u=(1+\sin (\pi(x+y-2 u t))) / 2 .
$$

The solution is smooth for $t<\frac{1}{\pi}$ and for $t \geq \frac{1}{\pi}$ contains shock waves. We first study the accuracy of the FC5 method for the Burgers' equation using both single-domain and multi-domain formulations for the smooth solution at time $t=0.15$. For the single-domain method, the number of points $N P=20^{2}, 40^{2}, 80^{2}$, and $160^{2}$, and for the multi-domain variant $N P=20$ and the number of domains are $N D=1,2^{2}, 4^{2}$, and $8^{2}$. In Fig. 3 , the maximum error is plotted versus the grid spacing. As is clear, the single- and multi-domain variants lead to comparable accuracy levels and convergence rates of 5.4 and 5.1, respectively, in agreement with the expectations.

We also consider the Burgers' equation at two later times $T=0.32$ and $T=0.64$, by which discontinuities in the solution have appeared. These results are produced by two different methods: the proposed hybrid FCWENO method with ninth-order accuracy for the WENO part and fifth-order of accuracy for the FC scheme, 


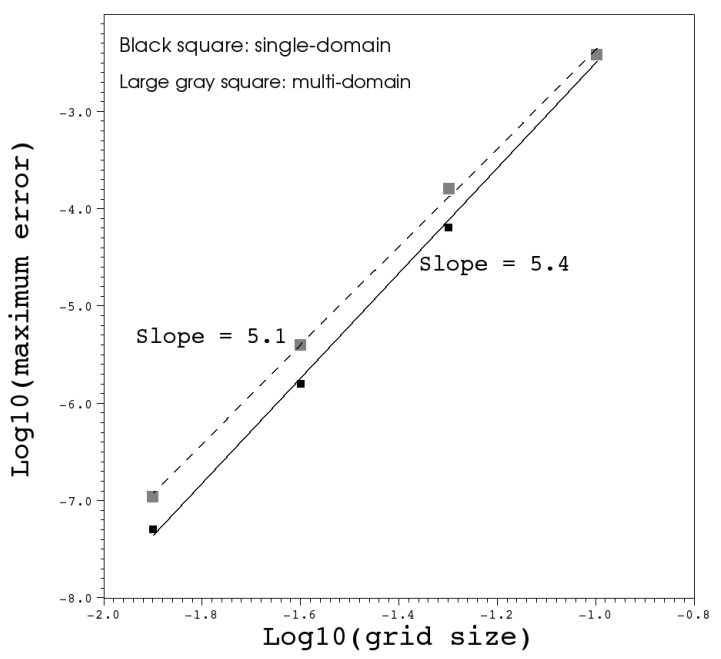

Figure 3: Convergence for the fifth-order Fourier continuation (FC5) method for the numerical solution of a 2D inviscid Burgers' equation in $[-1,1] \times[-1,1]$ with initial condition $u(0, x, y)=(1+\sin (\pi(x+y))) / 2$ and the final time $T=0.15$ with smooth exact solution using both multi-domain and single domain formulations. For the single-domain method, the number of points is $N P=20^{2}, 40^{2}, 80^{2}$, and $160^{2}$, while for the multi-domain $N P=20^{2}$ is fixed and the number of domains $N D=1,2^{2}, 4^{2}$, and $8^{2}$. For each data set, the best curve fit and its slope are also shown.

and a pure ninth-order WENO scheme. In the FC-WENO hybrid calculations, the multi-resolution analysis is carried out at every stage of the Runge-Kutta time stepping procedure with the order of the interpolating polynomial on the coarse grid $s=9$, and the tolerance $\epsilon \approx 10^{-3}$. In all the numerical experiments, we used time step size $\Delta t \approx 0.25 \Delta x / C$, where $\Delta x$ is the grid spacing and $C$ is the maximum wave speed.

In Fig. 4 (a), the FC-WENO computed solutions are shown in pseudocolor along with the sub-domains treated with a WENO scheme for $T=0.32$ and $N D=8^{2}$, while in Fig. 4 (b), the same data are shown for the later time $T=0.64$. Similar data with one level refinement are shown in Fig. 4 (c) and (d). As is clear from the figures, the WENO domains are restricted to the two regions containing shocks and as the number of domains increases, the percentage of the WENO domains decreases, which in turn results in higher speedup. The qualitative agreement of the computed solution with the exact solution are shown in Figs. 5 (a) and (b) along the line $x=y$ for $T=0.32$ and $T=0.64$, respectively. The solution of the pure WENO method is numerically identical to the that of the FC-WENO method. The quantitative comparison are shown in Table 1, where the $L_{1}$ norm error, convergence rate, the speedup over the pure WENO method and the average number of WENO domains (in percentage) are listed for $N P=20^{2}$ and different number of domains $N D=1,2^{2}, 4^{2}, 8^{2}, 16^{2}$. As evident from the table, a rate of convergence of approximately 1.0 is observed, verifying the theoretical estimate of the convergence rate for the schemes for discontinuous solutions. Also seen from the table, the percentage of the WENO domains indeed decreases by increasing the refinement and thus yielding better speedup.

Table 1: Convergence of the hybrid FC-WENO solution for a discontinuous solution of an inviscid Burgers' equation at $T=0.64$ with $N P=20^{2}$

\begin{tabular}{||c||c|c||c|c||}
\hline ND & $L_{1}$ error & Convergence rate & \# of WENO domains & Speedup over WENO solver \\
\hline 1 & $1.8 e-2$ & - & $80 \%$ & - \\
$2^{2}$ & $1.0 e-2$ & 0.85 & $68 \%$ & - \\
$4^{2}$ & $4.5 e-3$ & 1.2 & $43 \%$ & 1.5 \\
$8^{2}$ & $2.7 e-3$ & .74 & $19 \%$ & 2.5 \\
$16^{2}$ & $1.1 e-3$ & 1.3 & $9 \%$ & 3.1 \\
\hline
\end{tabular}



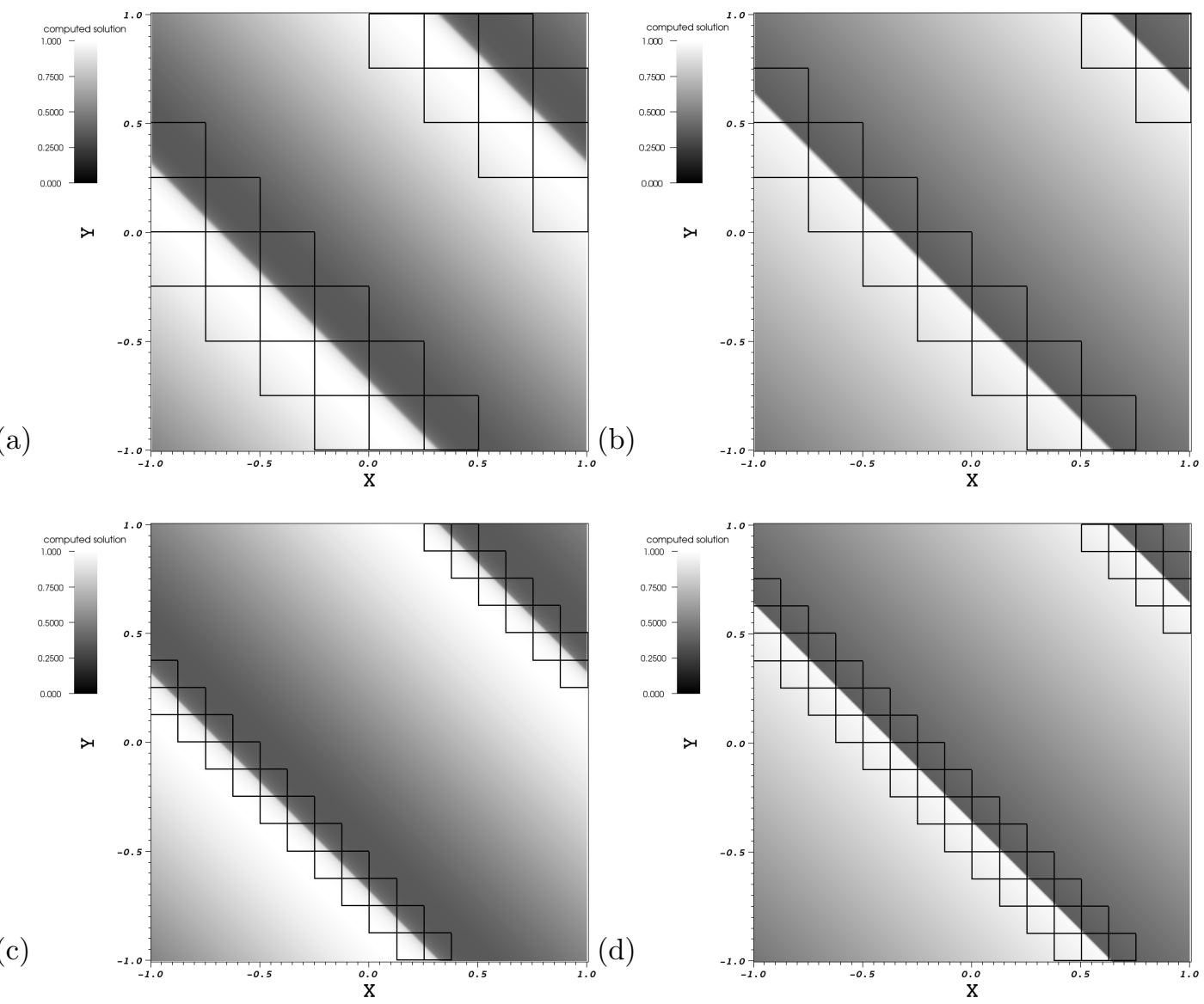

Figure 4: Pseudocolor of the inviscid Burgers' eq. solution computed using FC-WENO solver; (a) $T=0.32$ and $N D=8^{2} ;(\mathrm{b})$ $T=0.64$ and $N D=8^{2}$; (c) $T=0.32$ and $N D=16^{2}$; (d) $T=0.64$ and $N D=16^{2}$. Squares signify the sub-domains treated using WENO scheme.

\subsection{The 2D Euler system}

The two-dimensional Euler equations are given as

$$
\frac{\partial \mathbf{u}}{\partial t}+\frac{\partial \mathbf{F}(\mathbf{u})}{\partial x}+\frac{\partial \mathbf{G}(\mathbf{u})}{\partial y}=0
$$

where $\mathbf{u}=[\rho, \rho u, \rho v, \rho E], \mathbf{F}(\mathbf{u})=\left[\rho u, \rho u^{2}+p, \rho v^{2},(\rho E+p) u\right]$ and $\mathbf{G}(\mathbf{u})=\left[\rho v, \rho u^{2}, \rho v^{2}+p,(\rho E+p) v\right]$. Here, $\rho, E, p$, and $u$ and $v$ represent, respectively, the density, the total energy, the pressure, and velocities in the $x$ - and the $y$-direction. Equation (21) are subjected to appropriate initial and boundary conditions.

We consider first two test problems, both featuring the interaction of a right-moving shock wave with a plane entropy wave. The first problem, comprising a Mach 3 shock interaction with a small entropy wave having an angle $\theta_{0}=30^{\circ}$ with the shock front normal, which features supersonic flow behind the shock and allows for a quantitative comparison of our computed hybrid solutions with the pure WENO solutions and the analytical results obtained using a linear analysis of the Euler equations. The second problem involves a shock/entropy-wave interaction configuration with Mach 1.25 and a small entropy wave with $\theta_{0}=10^{\circ}$, featuring subsonic flows behind the shock and thus allowing the evaluation of the hybrid scheme in capturing the generated upstream-propagating acoustic waves and a comparison with another hybrid scheme arising from central difference-WENO schemes. Finally, we demonstrate the capability of our hybrid scheme in simulating the challenging Richtmyer-Meshkov instability problem and its good parallel performance on a distributed memory parallel architectures and on GPUs. 
(a)

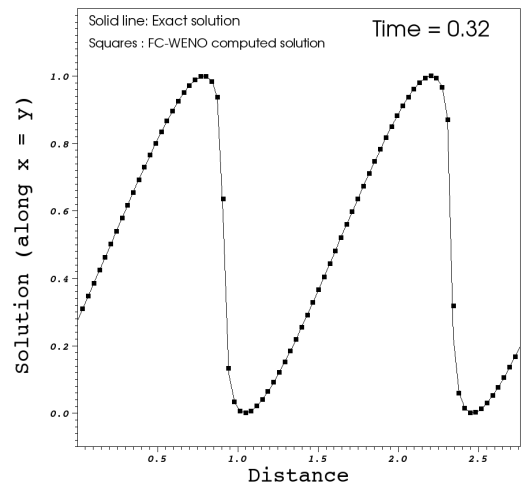

(b)

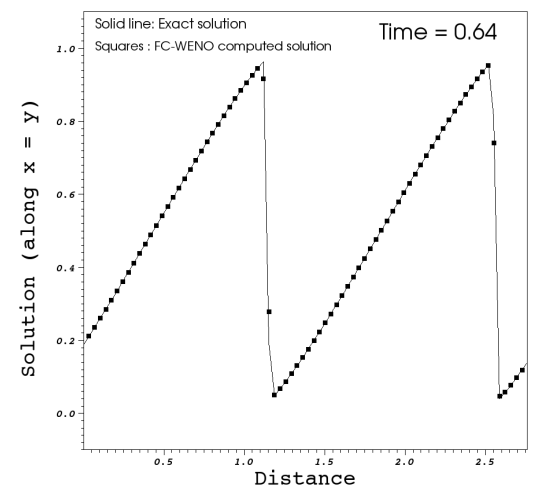

Figure 5: Computed FC-WENO solution of the 2D inviscid Burgers equations with $N P=20^{2}$ and $N D=4^{2}$ along with its exact solution. (a) $T=0.32$; (b) $T=0.64$.

\subsubsection{D Mach 3 Shock/small-entropy-wave interaction test}

To quantify the performance of the hybrid FC-WENO methods we consider a right-moving Mach 3 shock interacting with a very small entropy wave forming an angle $\theta_{0}=30^{\circ}$ with the normal vector to the shock front. The spatial domain is $[-10,10] \times\left[0, y^{*}\right]$, and the initial conditions are given by

$$
(\rho, u, v, p)(0, x, y)=\left\{\begin{aligned}
(3.857143,2.629369,0,10.33333) & x \leq-9.5, \\
(1,0,0,1) & -9.5 \leq x \leq-8.85, \\
\left(\operatorname { e x p } \left(-0.01 \sin \left(\kappa\left(\left(x-x_{0}\right) \cos \left(\theta_{0}\right)+y \sin \left(\theta_{0}\right)\right), 0,0,1\right)\right.\right. & x>-8.85,
\end{aligned}\right.
$$

where the wave number $\kappa=10, x_{0}=-8.85$ and $y^{*}=\frac{8 \pi}{\kappa \sin \left(\theta_{0}\right)}$ is defined to permit periodic boundary conditions in the $y$-direction. In the $x$-direction, boundary conditions are applied based on eq. (22). As a result of the interaction, based on the linear analysis [23], the entropy wave is reduced and transmitted with angle $\theta_{1}=26.6642$ and a pressure wave is generated with $\theta_{2}=8.5130$. Linear analysis also gives the amplitude of the pressure wave as 0.04378 [23], allowing the quantitative assessment of the accuracy of our hybrid method.

The Euler system is solved using both a pure WENO solver and the hybrid FC-WENO solver for the final time $T=5.0$. It is notable that the pure WENO solver does not include the multi-resolution analysis and the multi-domain overhead. For resolution $N D=48 \times 12$ and $N P=32^{2}$, the computed pressure and entropy using the hybrid technique are shown in Fig. 6 (a) and (b), where the corresponding pseudocolors are drawn. From the figures, the constant entropy and pressure contours form angles with the shock fronts closely matching $\theta_{1}$ and $\theta_{2}$ obtained from the linear analysis. Figs. 6 also demonstrate the multi-dimensional nature of this problem as entropy and acoustic waves propagate along different directions not parallel to the grid edges. Extraction of the pressure waves just behind the shock along $y=x \tan \left(\theta_{2}\right)$ and the density along $y=2.5$ are also presented in Fig. 7. The detailed views of the computed pressure and density, Figs. 7 top and bottom right, for three resolutions of $N D=16 \times 4, N D=32 \times 8$ and $N D=48 \times 12$ with $N P=32^{2}$ for all three cases clearly show that the solution convergence is achieved through the increasing resolution. To quantify this, Table 2 lists the errors in the acoustic wave amplitude right behind the shock for all three resolutions and for both hybrid FC-WENO and the pure WENO schemes. The convergence is clearly seen as errors of $9.3 \%, 2.3 \%$ and $0.8 \%$ are observed for the increasing resolution for both schemes. In the table, the CPU times and the average number of WENO domains are also reported, demonstrating the speedup of the hybrid scheme over the pure WENO solver.

\subsection{D Mach 1.25 Shock/small-entropy-wave interaction test}

We now compare our hybrid FC-WENO solver with other hybrid solver arising from the conjugation of the six- and eight-order central difference methods and the ninth-order WENO scheme (CD6-WENO9 and CD8-WENO9). The simplicity and low operation counts of central difference schemes suggest comparison 

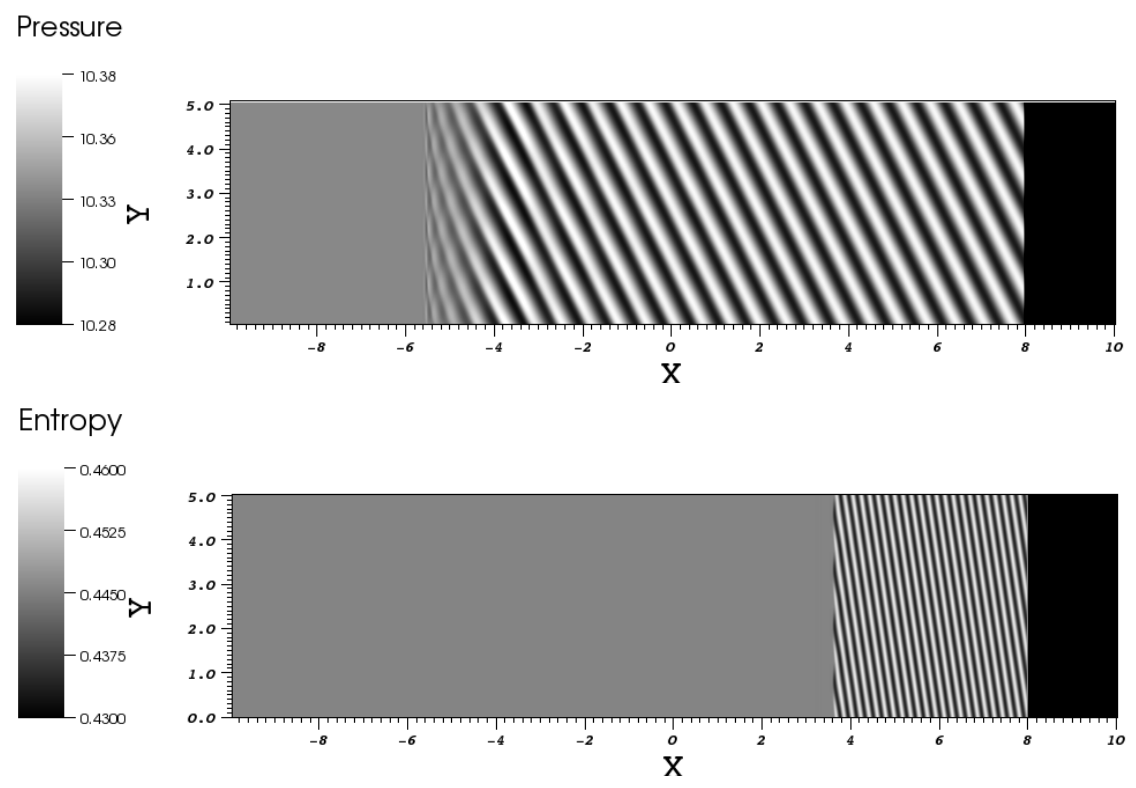

Figure 6: Pseudocolors of pressure (top) and entropy (bottom) behind a Mach 3 shock wave striking a small plane entropy wave with $\theta_{0}=30^{\circ}$ counterclockwise inclination from the horizontal direction, generating a pressure wave and transmitting the entropy wave (with reduced amplitude) at angles matching those from linear analysis [23].

Table 2: Shock interaction with small plain oblique entropy wave, Mach $=3, T=5, \theta_{0}=30^{\circ}, N P=32^{2}$.

\begin{tabular}{||c||c|c|c||}
\hline Method & Acoustic wave error & WENO domains \# & CPU time(s) \\
\hline FC-WENO, $N D=16 \times 4$ & $9.3 \%$ & $16 \%$ & $1.49 e+2$ \\
WENO, $N D=16 \times 4$ & $9.3 \%$ & $100 \%$ & $3.7 e+2$ \\
\hline FC-WENO, $N D=32 \times 8$ & $2.3 \%$ & $7.8 \%$ & $8.6 e+2$ \\
WENO, $N D=32 \times 8$ & $2.3 \%$ & $100 \%$ & $2.8 e+3$ \\
\hline FC-WENO, $N D=48 \times 12$ & $0.8 \%$ & $5.3 \%$ & $2.5 e+3$ \\
WENO, $N D=48 \times 12$ & $0.8 \%$ & $100 \%$ & $8.8 e+3$ \\
\hline
\end{tabular}

of the FC5-WENO9 with not only the CD6-WENO9 (which has comparable order of accuracy), but with even higher order CD8-WENO9. However, as shown below, owning to its almost dispersionless character, the FC5-WENO9 outperforms both CD6-WENO9 and CD8-WENO9 for the cost (CPU time) of achieving a given accuracy level. The test problem is the shock small entropy interaction problem. A Mach $M=1.25$ shock wave moving rightward interacting with an oblique entropy wave. The density disturbance of the entropy wave are set as

$$
\rho=\exp \left(-0.01 \sin \left(\kappa\left(\left(x-x_{0}\right) \cos \left(\theta_{0}\right)+y \sin \left(\theta_{0}\right)\right)\right)\right.
$$

where $\theta_{0}=10^{\circ}$ being the counterclock-wise angle between the entropy wave and the normal to the unperturbed shock front, $x_{0}=11$, and the wave number $\kappa=15$. The computational domain is chosen to be a rectangle with $[-10,54] \times\left[0, y^{*}\right]$, where the width of rectangle is chosen to allow periodic boundary conditions in the $y$-direction, $y^{*}=\frac{2 \pi}{\kappa \sin \left(\theta_{0}\right)}=2.4122$. The Euler system with the above setting is solved using both FCWENO and CD-WENO schemes. Three simulation runs were carried out: FC-WENO with high-resolution of $N P=96^{2}$ and $N D=64 \times 4$, and FC-WENO and CD-WENO both with (one level) lower resolution of $N P=96^{2}$ and $N D=32 \times 2$. The relative density $\left(\rho-\rho_{0}\right.$, where $\rho_{0}=1.42857$ is the density behind the shock wave in the absence of the entropy wave) along the line $y=1.0$, obtained using the high-resolution computation are shown in Fig. 8 (top left). Two distinctive regions of acoustic waves far from the shock and the region consisting of entropy, vorticity and acoustic waves immediately behind the shock are evident. Since the amplitude of the acoustic wave are much smaller than the entropy wave (approximately 10\% of the entropy wave) any insignificant error in the composite region behind the shock with a mix of various waves can pollute the computation of pure acoustic waves propagating upstream. A zoomed-in view, centered at 

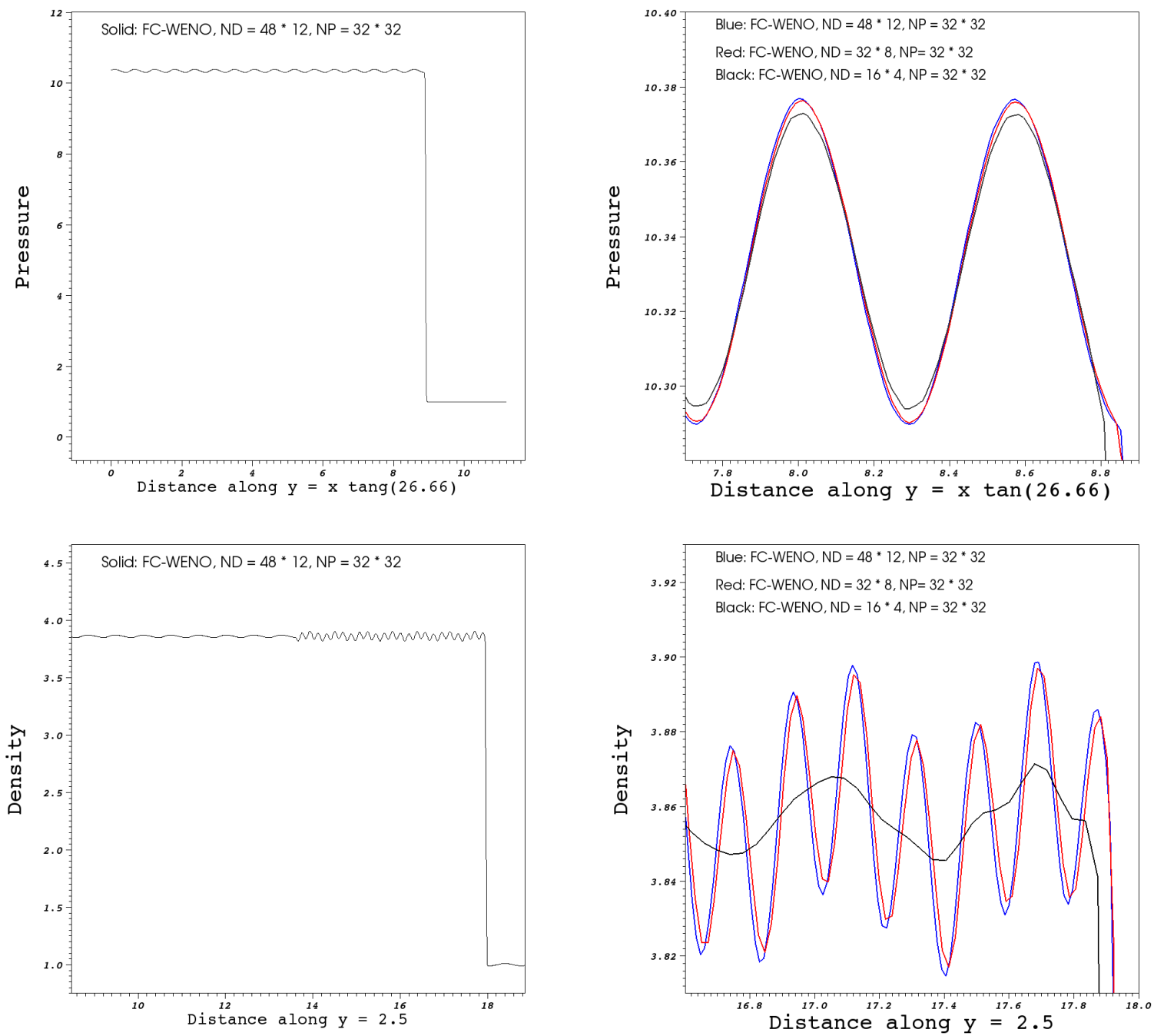

Figure 7: Overall view of the computed solution of the Mach 3 shock/small-entropy-wave interaction extracted along a particular line at final time $T=5.0$, demonstrating the overall profile and the convergence under increasing resolution. Top left, pressure profile along $y=x \tan (26.66)$; top right, detailed view of the pressure right behind the shock for solutions computed using three different resolutions; bottom left, density profile along $y=2.5$; bottom right, detailed view of the computed density for three different resolutions.

the transition region, of the solution along with the graph of errors in density arising from one-level coarser grid for FC5-WENO9, CD6-WENO9 and CD8-WENO9 solvers are shown in Fig. 8 (top right). A further zoomed-in view of the same data is shown in Fig. 8 (bottom). As is evident from the figures, the FC-WENO approach is significantly more accurate than the two CD-WENO schemes in the tail of the purely acoustic wave region. For qualitative comparison, the $L_{1}$ norm errors for the two schemes along with the total CPU times are shown in Table 3 . The $L_{1}$ errors are computed as

$$
L_{1}(\text { error })_{\left[x_{1}, x_{2}\right], y=1}=\frac{\int_{x_{1}}^{x_{2}}\left|\rho-\rho^{*}\right| d x}{\int_{x_{1}}^{x_{2}}\left|\rho^{*}-\rho_{0}\right| d x},
$$

where $\rho_{0}=1.42857$ is the density behind the normal shock with $M=1.25$ and $\rho^{*}$ is the high-resolution computed density. (We consider this solution as the 'exact density', since it results in an acoustic wave with amplitude of 0.002000 changing less than $1 \%$ under grid refinement and closely matching that of linear theory prediction, 0.001975 [23]). The $L_{1}$ errors are computed in $\left[x_{1}=21.3, x_{2}=22.2\right]$ (the purely acoustic region) and in $\left[x_{1}=22.2, x_{2}=23.2\right]$. As reported in the table, while both hybrid techniques result in less than $10 \%$ error in the second region, in the first region, the FC-WENO solver yields significantly lower error, less than $5 \%$, as compared to the $36 \%$ and $13.4 \%$ errors of the CD6-WENO9 and CD8-WENO9 schemes. Since both FC-WENO and CD-WENO schemes requires comparable total CPU times (FC-WENO requires roughly $10 \%$ higher), the FC-WENO emerges as a significantly more efficient solver. Even accuracy of the one-level 
Table 3: Shock interaction with small plain oblique entropy wave, Mach $=1.25, T=39.5, N P=96^{2}$.

\begin{tabular}{||c||c|c|c||} 
Method & $L_{1}$ (error) $[21.3,22.2], y=1$ & $L_{1}(\text { error })_{[22.2,23.2], y=1}$ & CPU time $(\mathrm{s})$ \\
\hline CD6-WENO9, $N D=32 \times 2$ & $36 \%$ & $8.5 \%$ & $5.8 e+3$ \\
CD8-WENO9, $N D=32 \times 2$ & $13.4 \%$ & $8.5 \%$ & $6.0 e+3$ \\
FC5-WEN09, $N D=32 \times 2$ & $4.8 \%$ & $8.5 \%$ & $6.2 e+3$ \\
\hline CD6-WENO9, $N D=64 \times 4$ & $18.7 \%$ & $1.4 \%$ & $4.6 e+4$ \\
FC5-WENO9, $N D=64 \times 4$ & - & - & $5.1 e+4$ \\
\hline
\end{tabular}

refinement of the CD6-WENO9 solution contains 18.7\% error at a cost of approximately eight-fold higher CPU time (Table 3). The large error of the CD-WENO scheme is primarily due to the inherent dispersion errors of the central difference methods. As stated earlier, the high-level of accuracy of the FC-WENO solver, on the other hand, is due to the almost dispersion-free character of the Fourier continuation method.

\subsection{A 2D Richtmyer-Meshkov instability simulation on parallel CPUs and on a single GPU}

The multi-domain framework has been implemented with ease and tested with success on parallel processors using the Message Passing Interface (MPI) as well as on many core Graphics Processing Units (GPUs). The performance of the hybrid solver for simulating early stages of a Richtmyer-Meshkov instability problem have been investigated. As an initial test case we consider a Mach 4.46 shock interacting with a material interface of two gases Xenon and Argon in a spatial domain of $[12,3.6]$ similar to that in [12].

Figure 9 (a) depicts the density at the physical time of $\approx 128 \mu \mathrm{s}$ for resolutions of a $(64 \times 20)$ array of sub-domains and the number of points per domain of $N P=32^{2}$. Figure 9 (b) also shows the sub-domains discretized using the WENO solver. Clearly, the use of WENO scheme is restricted to regions containing discontinuous solutions, hence decreasing the overall computational cost. To illustrate the efficiency of the hybrid solver over the pure WENO solver, Table 4 lists the wall clock time in seconds for the same RichtmyerMeshkov instability problem computed using the hybrid FC-WENO solver for three resolutions $(32 \times 10)$, $(64 \times 20)$ and $(128 \times 40)$ sub-domains. As is evident, the hybrid solver is five to seven times faster than the pure WENO solver.

Our parallel strategy on multiple processors is based on distributing the equal portions of the number of sub-domains, obtained from partitioning the sub-domain grid along the $y$-direction, among the processors. As shown in Table 4, the hybrid solver displays great parallel performance, $96 \%$ or higher efficiency, illustrating the minimal inter-processors communications required which is in turn crucial for the three-dimensional computations. It is notable that this particular domain partitioning is highly effective for cases with a plane shock wave occupying the entire length of the domain in one of the directions (in this case $y$-direction). For more general problems, this strategy may yield some load unbalance among processors and thus results in reduced parallel efficiency. How large the impact would be in the context of large three dimensional calculations with large number of domains is a subject of future work.

Table 4: The hybrid FC-WENO solver performance in simulating Richtmyer-Meshkov instability problem

\begin{tabular}{||c|c|c|c|c||}
\hline ND & \# of processor & CPU time $(\mathrm{s})$ & Speedup over WENO solver & Parallel Efficiency \\
\hline $32 \times 10$ & 1 & $4.2 \times 10^{3}$ & 5.6 & - \\
$32 \times 10$ & 10 & $4.3 \times 10^{2}$ & 5.5 & $98 \%$ \\
\hline $64 \times 20$ & 1 & $2.7 \times 10^{4}$ & 6.4 & - \\
$64 \times 20$ & 20 & $1.4 \times 10^{3}$ & 6.2 & $96 \%$ \\
\hline $128 \times 40$ & 1 & $9.6 \times 10^{4}$ & 7.0 & - \\
$128 \times 40$ & 40 & $2.5 \times 10^{3}$ & 6.7 & $96 \%$ \\
\hline
\end{tabular}

We also implemented the hybrid solver on Graphics Processing Units (GPUs). The multi-domain formulation lends itself naturally to the thread block parallel processing of the data in the CUDA programming framework [42] in which we simply map each subdomain into one GPU block. Here, we highlight some important steps on GPU in order to achieve reasonable efficiency. All computations are carried out on the GPU and the data transfer between the CPU and GPU are limited to only one round, before and after the time stepping stage. In the current implementations the data is stored in the global memory and the shared memory of the GPU is utilized to minimize the memory latency. 

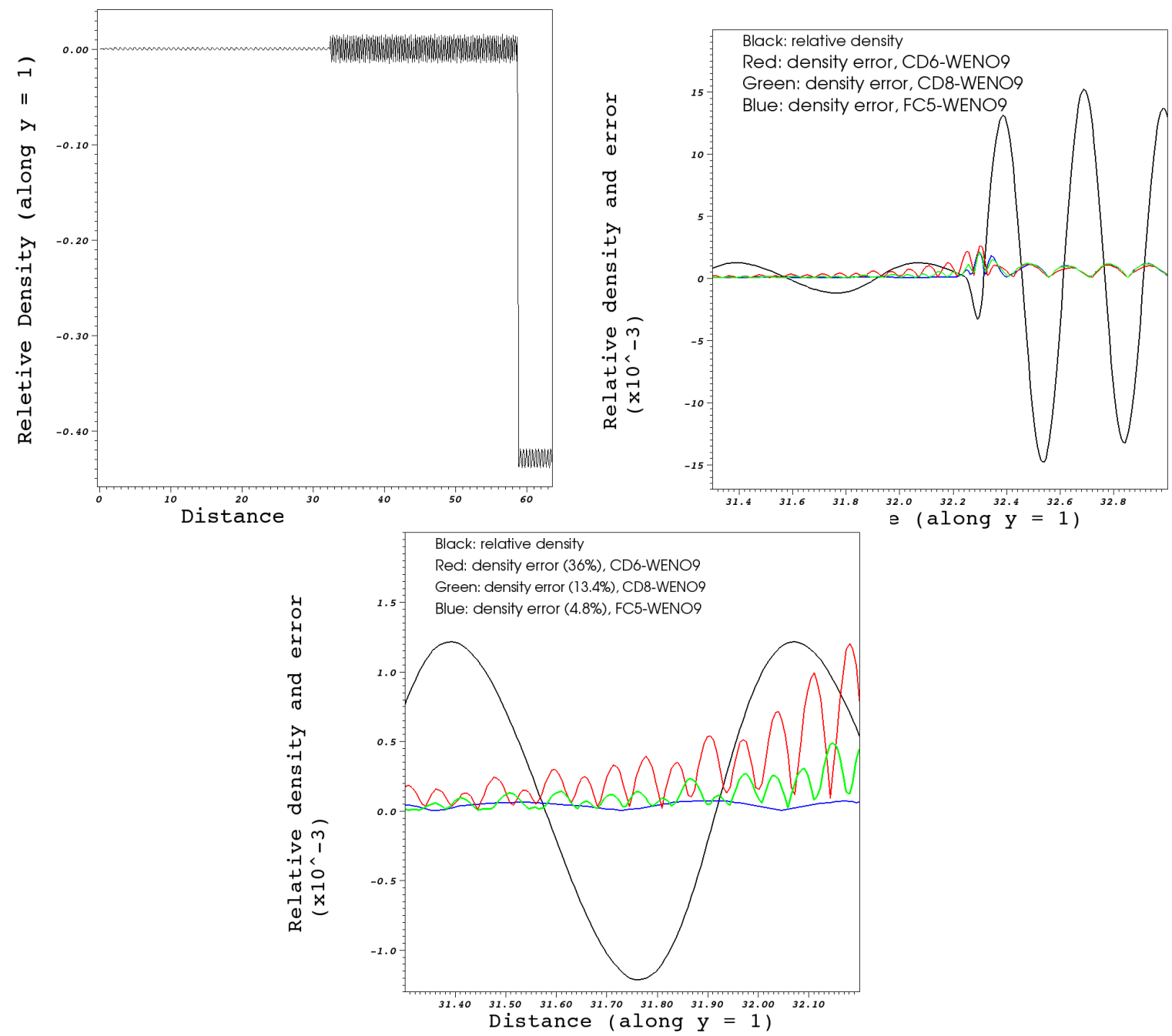

Figure 8: Computed FC-WENO density and errors of the 2D Euler equations for Mach 1.25 shock wave striking a small oblique entropy wave with $N P=64 \times 4$ and $N P=96^{2}$; top left, the overall view showing the pure acoustic region and the composite region containing all three characteristic waves; top right, detailed view of the computed density errors in the transition region for C6D-WENO9, CD8-WENO9 and FC5-WENO9 schemes; bottom, a zoommed-in view of the tail of the acoustic wave region, demonstrating the superior accuracy of the FC-WENO scheme over both CD6-WENO9 and CD8-WENO9.

Since the WENO kernel is relatively easy to implement efficiently on GPUs the key to reasonable performance for the hybrid schemes is to find the right strategy for the FC kernel on GPU. There are two major concerns: efficient FFT routines for local FC derivative computations, with typical small sizes of $N P=32$ or 96, are not readily available in the GPU implementation since the CUDA version of FFT, CUFFT, is a global function CPU and optimized only for data of very large sizes; simply aggregating the Fourier subdomains and applying CUFFT naively will increase memory traffic on GPU due to the dynamic nature of the hybrid schemes. Therefore, we use an alternative approach for derivative calculations based on matrix operations [21]. Recall that since the differentiation matrix is circulant, it is enough to store one row of the matrix. Taking advantage of this property will not only lessen the pressure of the shared memory, but also impact the memory bandwith significantly. In addition, this matrix-based approach can avoid the extra computation by turning off the derivative computation on the extension points, something that can not be avoided by FFT-based approach.

For the same RMI simulation with $N P=32$, the speedup of the GPU computation of the hybrid and the pure WENO schemes over the CPU computations using hybrid FC-WENO scheme for various number of domains are shown in Fig. 10 (a). All computations are carried out in double precision floating point 

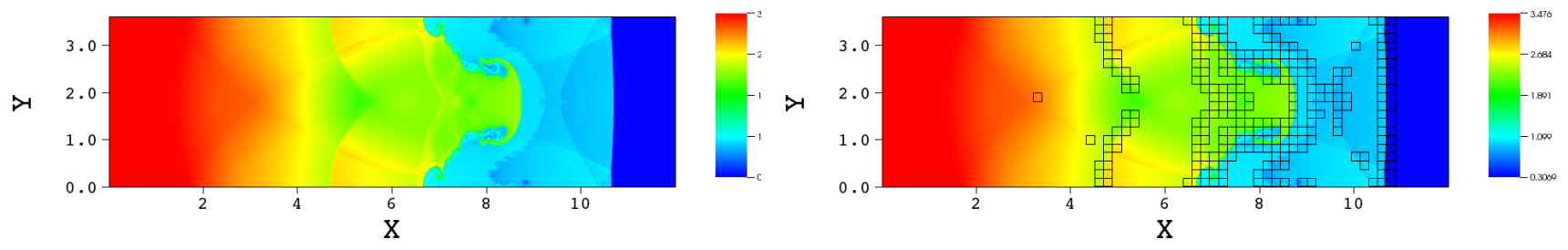

Figure 9: (a) Density contours for a Richtmyer-Meshkov instability at physical time of $128 \mu \mathrm{s}$ in which a shock wave with Mach of 4.46 interacting with a single-mode disturbed interface of Xenon and Argon gases; (b) WENO domains are also shown using the square symbols.

arithmetics on a Nvidia Tesla C2050 (Fermi) GPU and the AMD Phenom(tm) 9850 Quad-Core @1.25 GHz CPU. As is clear, the hybrid GPU implementation achieves five- to six-fold speedup over the hybrid CPU. Fig. 10 (b) shows the ratio of the computation time of the hybrid solver over the pure WENO solver for both CPU and GPU calculations. Clearly, similar ratios are obtained in both architectures highlighting that potential gain of the hybrid schemes over the WENO solver on CPU computations extends uniformly to the GPU computations.

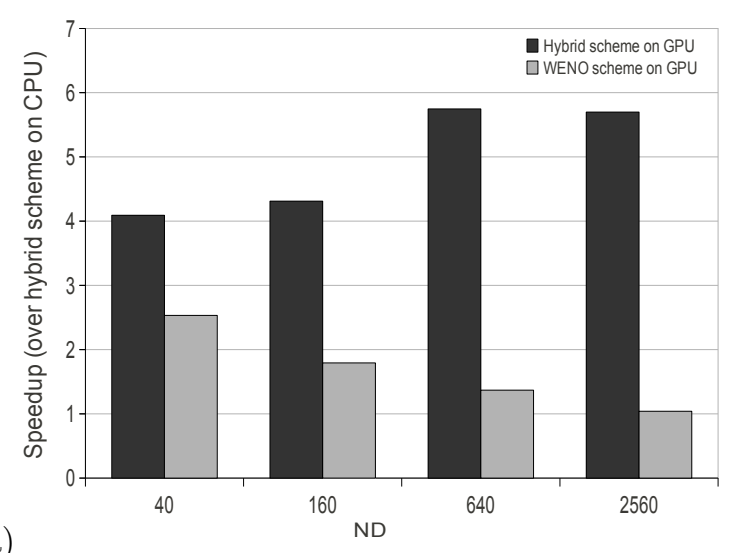

(a)

(a) ND (b)

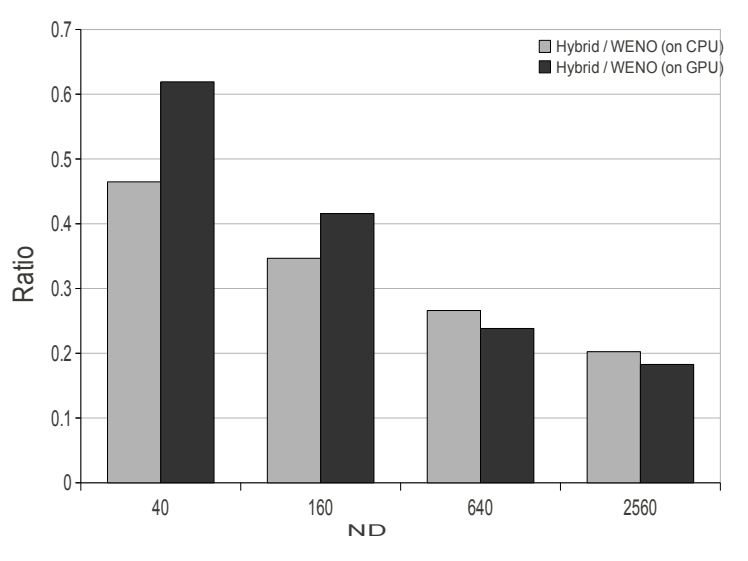

Figure 10: (a) Speedup of the hybrid and WENO computations of RMI problem on a single GPU over the hybrid computations on a single CPU; (b) ratio of the time taken for hybrid computations over the WENO computations on both a single GPU and a single CPU, demonstrating similar gain for hybrid solver over the pure WENO solver on both architectures.

\section{Conclusions}

We have introduced a high-order method based on the hybridization of the Fourier continuation method and WENO finite difference discretization methods for the solution of two dimensional non-linear conservation laws. The hybrid strategy is based on a multi-domain formulation with a smoothness detection procedure that effectively flags the domains containing discontinuities. These are treated by a WENO scheme and those with smooth solutions are discretized using a Fourier continuation method. For applications with isolated discontinuities in space and time, this strategy offers significant advantages over the pure WENO methods, since the majority of the domains are discretized using the FC method which is considerably less expensive than the WENO reconstruction, yet offers a highly efficient and accurate procedure for complex but smooth portions of the solution. The multi-domain strategy facilitates simple and efficient implementation of the solver on contemporary parallel processors using message passing interface for inter-processor communications as well as on emerging many core Graphics Processing Units using CUDA programming. 
The stability, accuracy and efficiency of the hybrid FC-WENO solver is illustrated using various challenging two-dimensional test problems. In a Mach 3 shock wave interaction with an oblique small entropy wave, the hybrid scheme provides a solution in agreement with the linear stability analysis and matching that of the pure WENO solver, but with significant savings in the required computational time. Owing to its almost dispersion-less character, the hybrid FC-WENO outperforms, by at least an order of magnitude, an alternative hybrid scheme, the central difference-WENO, in solving Mach 1.25 shock wave interaction with a small entropy waves which features the generated acoustic wave propagating upstream. The excellent performance of the hybrid schemes for simulating the challenging two-dimensional Richtmyer-Meshkov instability is also demonstrated. For the same problem, the parallel solution of the solver on multiple CPUs achieves excellent scaling and the single GPU computations, in double precision, yields a six-fold speedup over the single CPU computations.

Although the current state of the hybrid solver already offers significant advantages over the existing alternatives, be that the pure WENO or the hybrid CD-WENO solvers, further capabilities can be build into the hybrid framework to enable simulation of complex scientific and engineering problems. In particular, high-fidelity modeling of compressible multi-phase flows with shock waves, or high-amplitude ultrasound waves, and bubbles featuring complex, nonlinear, discontinuous structures, necessitates even higher level of resolution along the shock fronts and inter-phase boundaries. The required higher resolution can be achieved through even higher order WENO schemes (higher than 9) or through local grid refinements, the two strategies that we are currently pursuing. Interface capturing schemes for gas-liquid interfaces require cell-averaging formulations, essential for avoiding spurious oscillations in the computed solutions using the point-wise approach [40, 41]. Such extensions to cell-averaging formulations as well as to complex geometries are also ongoing.

\section{Acknowledgments}

This work was financially supported by US Department of Energy, under Contract DE-FG02-98ER25346. The authors appreciate insightful discussions with Oscar Bruno and Nathan Albin during the initial phase of this work.

\section{References}

[1] N. Albin and O. P. Bruno, A spectral FC solver for the compressible Navier-Stokes equations in general domains I: explicit time-stepping, J. Comput. Phys. 230(2011), 6248-6270.

[2] J. P. Boyd, A comparison of numerical algorithms for Fourier extension of the first, second, and third kinds, J. Comput. Phys. 178 (2002) 118-160.

[3] J. P. Boyd and J. R. Ong, Exponentially-convergent strategies for defeating the Runge phenomenon for the approximation of non-periodic functions, part I: single-interval schemes, Commun. Comput. Phys., 5 (2009) 484-497.

[4] J. P. Boyd and J. R. Ong, Exponentially-convergent strategies for defeating the Runge Phenomenon for the approximation of non-periodic functions, part two: Multi-interval polynomial schemes and multidomain Chebyshev interpolation. J. Appl. Numer. Math., 61 (2011) 460-472.

[5] O. P. Bruno, Y. Han and M. M. Pohlman, Accurate, high-order representation of complex threedimensional surfaces via Fourier continuation analysis, J. Comput. Phys. 227 (2007) 1094-1125.

[6] O. P. Bruno and M. Lyon, High-order unconditionally stable FC-AD solvers for general smooth domains I. Basic elements, J. Comput. Phys. 229 (2010) 2009-2033.

[7] M. Lyon and O. P. Bruno, High-order unconditionally stable FC-AD solvers for general smooth domains II. Elliptic, parabolic and hyperbolic PDEs; theoretical considerations, J. Comput. Phys. 229 (2010) 3358-3381. 
[8] L. N. Trefethen and D. Bau, III, Numerical Linear Algebra, Soc. for Industr. and Appl. Math., Philadelphia, 1997.

[9] A. Harten, Adaptive multiresolution schemes for shock computations, J. Comput. Phys. 115 (1994) 319-338.

[10] B. Costa and W. S. Don, Multi-domain hybrid spectral-WENO methods for hyperbolic conservation laws, J. Comput. Phys. 224 (2007) 3358-3381.

[11] B. Costa and W. S. Don, High order hybrid central-WENO finite difference scheme for conservation laws, J. Comput. Appl. Math. 204 (2007) 209-218.

[12] B. Costa, W. Don, D. Gottlieb and R. Sendersky, Two-dimensional multi-domain hybrid spectral- WENO methods for conservation laws, Commun. Comput. Phys., 1 (2006) 550-577.

[13] G.S. Jiang, C.W. Shu, Efficient implementation of weighted ENO schemes, J. Comput. Phys. 126 (1996) $202-228$

[14] C.-W. Shu, Essentially non-oscillatory and weighted essentially non-oscillatory schemes for hyperbolic conservation laws, in: A. Quarteroni (Ed.), Advanced Numerical Approximation of Nonlinear Hyperbolic Equations, Lecture Notes in Mathematics, vol 1697, Springer, Cetraro, Italy, (1997) 325.

[15] C. Shu, High order weighted essentially nonoscillatory schemes for convection dominated problems, SIAM Review, 51 (2009) 82-126.

[16] D. S. Balsara and C. Shu, Monotonicity preserving weighted essentially non-oscillatory schemes with increasingly high order of accuracy, J. Comput. Phys. 160 (2000) 405-452.

[17] C.-W. Shu and S. Osher, Efficient implementation of essentially non-oscillatory shock capturing schemes, J. Comput. Phys., 77 (1988), 439-471.

[18] X-D. Liu, S. Osher, and T. Chan, Weighted essentially non-oscillatory schemes, J. Comput. Phys. 115, (1994) 200-212.

[19] R. Borges, M. Carmona a, B. Costa and W. S. Don, An improved weighted essentially non-oscillatory scheme for hyperbolic conservation laws, J. Comput. Phys. 227, (2007) 3191-3211.

[20] D. Gottlieb and J. S. Hesthaven, Spectral methods for hyperbolic problems, J. Comput. Appl. Math. 128 (2001) 83-131.

[21] J. S. Hesthaven, S. Gottlieb and D. Gottlieb, Spectral Methods for Time-Dependent Problems, Cambridge Monographs on Applied and Computational Mathematics 21. Cambridge University Press, Cambridge, UK. X+274 pages, January 2007.

[22] M. K. Morkovin, Note on the assessment of flow disturbances at a blunt body traveling at supersonic speeds owing to flow disturbance in free stream. J. Appl. Mech. 27 (1960) 223-229.

[23] J. F. McKenzie and K. O. Westphal, Interaction of linear waves with oblique shock waves, Phys. Fluids 11 (1968) 2350-2362.

[24] J. Shi, Y. Zhang and C. Shu, Resolution of high order WENO schemes for complicated flow structures, J. Comput. Phys. 186 (2003) 690-696.

[25] M. Latini, O. Schilling and W. Don, Effects of WENO flux reconstruction order and spatial resolution on reshocked two-dimensional Richtmyer-Meshkov instability, J. Comput. Phys. 221 (2007) 805-836.

[26] O. Schilling and M. Latini, High-order WENO simulations of three-dimensional reshocked RichtmyerMeshkov instability to late times: dynamics, dependence on initial conditions, and comparisons to experimental data, Acta Math. Scientia 30 (2010), 595-620. 
[27] I.A. Babuska and S.A. Sauter. Is the pollution of the FEM avoidable for the Helmholtz equation considering high wave numbers? SIAM J. Numer. Anal., 34 (1997) 2392-2423.

[28] L. Jameson. High order schemes for resolving waves: Number of points per wavelength. J. Sci. Comput., 15 (2000) 417-433.

[29] S. Gottlieb and C. Shu, Total variation diminishing Runge-Kutta schemes, Math. Comp. 67 (1998), 73-85.

[30] G. Dahlquist and A. Bjork Numerical Methods, Prentice Hall, Englewood Clis, 1974.

[31] B. Gustafsson, High Order Difference Methods for Time Dependent PDE, Springer, 2008.

[32] C.G. Canuto, M. Y. Hussaini, A. Quarteroni, T.A. Zang, Spectral Methods: Evolution to Complex Geometries and Applications to Fluid Dynamics, Springer, 2007.

[33] D. A. Kopriva and J. H. Kolias, A conservative staggered-grid Chebyshev multidomain method for compressible flows, J. Comput. Phys. 125 (1996) 244-261.

[34] D. Funaro and D. Gottlieb, A new method of imposing boundary conditions in pseudospectral approximations of hyperbolic equations, Math. Comput., 51 (1988) 599-613.

[35] D. Gottlieb, M. Y. Hussaini and S. A. Orszag, Theory and application of spectral methods, in Spectral Methods for Partial Differential Equations, edited by R. G. Voigt, D. Gottlieb, M. Y. Hussaini, SIAM, 1984, Philadelphia.

[36] E. Johnsen and T. Colonius, Numerical simulations of non-spherical bubble collapse, J. Fluid Mech. 629 (2009) 231-262.

[37] G. A. Gerolymos, D. Senechal and I. Vallet, Very High Order WENO Schemes, J. Comput. Phys. 228 (2009) 84818524.

[38] M. Castro, B. Costa and W. S. Don, High order weighted essentially non-oscillatory WENO-Z schemes for hyperbolic conservation laws, J. Comput. Phys. 230 (2011) 17661792.

[39] K. Shahbazi, N. Albin, O. Bruno and J. Hesthaven, Multi-domain Fourier-continuation/WENO hybrid solver for conservation laws, J. Comput. Phys. 230 (2011) 8779-8796.

[40] S. Karni, Multicomponent flow calculations by a consistent primitive algorithm, J. Comput. Phys. 112 (1994) 31-43.

[41] R. Abgrall, How to prevent pressure oscillations in multicomponent flow calculations: a quasi conservative approach, J. Comput. Phys. 125 (1996) 150-160.

[42] http://docs.nvidia.com/cuda/cuda-c-programming-guide/index.html 